\title{
RESEARCH
}

\section{PREDICTIVE EFFECTS OF FIRST ERYTHROCYTE AND THROMBOCYTE VOLUME INDICES ON MORTALITY OF GERIATRIC PATIENTS WITH SEPSIS HOSPITALIZED IN INTENSIVE CARE UNITS}

Turkish Journal of Geriatrics
DOI: $10.31086 /$ tigeri.2021.208

2021; 24(2): 134-142

- Esra ÇAKIR ${ }^{1}$

- |şII ÖZKOÇAK TURAN ${ }^{1}$

\section{CORRESPONDANCE}

\section{Esra ÇAKIR}

Health Sciences University, Ankara Numune Education and Research Hospital, Anesthesiology and Clinical of Critical Care Ankara, Turkey

\section{Phone: +90541 8307079}

e-mail: pavulonmouse@hotmail.com

Received: Jan 04, 2021

Accepted: Apr 06, 2021

\section{${ }^{1}$ Health Sciences University, Ankara}

Numune Education and Research Hospital Anesthesiology and Clinical of Critical Care, Ankara , Turkey

\section{Abstract}

Introduction: Sepsis is a common reason for hospitalization in the intensive care unit and a cause of increased mortality, especially among elderly patients. We aimed to evaluate the relationship between erythrocyte and platelet indices and mortality of elderly patients admitted to the intensive care unit due to sepsis.

Materials and Methods: This study was retrospectively performed in patients with $\geq 65$ years of sepsis who were admitted to the intensive care unit. Hemoglobin, mean corpuscular volume, red cell distribution width (RDW), mean platelet volume (MPV), platelet distribution width (PDW), white blood cell and platelet count results, and demographic and clinical features were compared between survivors and non-survivors.

Results: RDW, MPV, PDW, and C-reactive protein were found to be significantly higher in the non-survivors group than survivor group $(p<0.001)$. We also determined that platelet and hemoglobin levels were significantly lower in the non-survivors group than survivor group $(p<0.001)$. In the estimation of mortality, the cut-off value was $\geq 16.3 \%$ for RDW, $\geq 10 \mathrm{fL}$ for MPV, $\geq 11.6 \%$ for PDW, $\leq 281 \times 10^{3} / \mathrm{\mu L}$ for platelets, $\leq 9.8 \mathrm{~g} / \mathrm{dL}$ for hemoglobin, and $\geq 86 \mathrm{mg} / \mathrm{L}$ ( $p=0.0001)$ for $\mathrm{C}$-reactive protein.

Conclusion: Increasing counts for red cell distribution width, mean platelet volume, and platelet distribution width and decreasing counts for platelets and hemoglobin for elderly patients admitted to the intensive care unit with sepsis may alert intensive care specialists to estimate mortality.

Keywords: Aged; Blood Platelets; Intensive care units; Erythrocyte Indices; Mean platelet volume; Sepsis. 


\section{INTRODUCTION}

The fastest growing age group in the world is $\geq 80$-year-old group, which increases by about 3.8\% per year (1). According to data from the Turkish Statistical Institute, the geriatric population increased from $7.5 \%$ in 2012 to $8.8 \%$ in 2018 (2). In many countries, the average age and life expectancy of the population has also increased. Old age is associated with chronic disease and functional impairment, and more elderly patients are being admitted to the intensive care unit (ICU) $(1,2)$. In addition, about half of ICU days are utilized by patients over 65 years old $(3,4)$.

Sepsis is a systemic inflammatory host response to infection that can lead to acute organ dysfunction and consists of clinical, hemodynamic, and biochemical components (4). Unfortunately, sepsis is a fatal condition with a $20-40 \%$ mortality rate and contributes to approximately $20 \%$ of in-hospital deaths (5). Elderly patients are more prone to infections due to the effects of aging, various associated comorbidities, the use of invasive devices, and interventions, so the occurrence of sepsis increases with age (1). In addition, the probability of death due to sepsis is higher in elderly patients (4). There is limited epidemiological information about the effect, incidence, and outcomes of sepsis in elderly patients (1). As our population ages, it is expected that the incidences of elderly patients with sepsis will increase and understanding sepsis mortality in this population and how sepsis effects their functional status becomes increasingly important (4). Therefore, it is very important to determine the mortality markers to improve the treatment of elderly patients with high mortality sepsis (6).

Sepsis is an important disease affecting almost all systems and the hematological system specifically is affected negatively (7). Sepsis causes various changes to red blood cell and platelet counts. These changes include indices, such as mean corpuscular volume (MCV), red cell distribution width (RDW), mean platelet volume (MPV), and platelet distribution width (PDW) in the complete blood count $(7,8)$. The number of studies investigating the clinical results of the changes in these parameters, especially in elderly patients with sepsis, is very limited. Therefore, the primary purpose of our study is to determine the relationship of erythrocyte and platelet volume indices with the mortality of elderly patients hospitalized in the ICU due to sepsis.

\section{MATERIALS AND METHODS}

\section{Ethical approval and study protocol}

Our ICU is a level 3 facility for all patients over 18 years of age. Our study was planned retrospectively in patients $\geq 65$ years old who had undergone sepsis in our ICU and includes patients hospitalized between March 2017 and March 2019. Patients who were primarily out of sepsis (trauma, intoxication, metabolic disorders, myocardial infarction, etc.) and $<65$ years of age were excluded from the study. The local clinical ethics committee approved the research before the study commenced. Our study was carried out in accordance with the principles of the Helsinki Declaration.

\section{Data sources}

Sepsis diagnosis for inclusion in the study was made according to Third International Consensus Definitions criteria, which depends on the clinical and laboratory findings of the patients (9). All registered patients were treated according to the International Guidelines for Management of Sepsis and Septic Shock after being admitted to the ICU (10). Age, gender, comorbid disease number (diabetes mellitus, hypertension, malignancy / immune deficien$c y$, respiratory system disease, heart disease, renal disease, central nervous system disease), acute physiology and chronic health evaluation (APACHE) II score, sepsis-related organ failure assessment (SOFA) score, duration of mechanical ventilation (MV), hospital stay, C-reactive protein (CRP), complete blood count $(\mathrm{CBC})$ analysis, blood cultures, and mortality data were recorded $(11,12)$. 


\section{Blood sampling for serum levels for CRP and CBC analysis}

Venous blood samples were taken in tubes containing ethylenediamine tetra-acetic acid from patients with sepsis during ICU hospitalization. Hemoglobin, MCV, RDW, MPV, PDW, and white blood cell (WBC) and platelet counts were measured twice-daily with a calibrated Cell-Dyn 3700 automated hemocytometer (Abbott, Abbott Park, IL, USA). Serum CRP concentrations were measured on a Roche Modular $\mathrm{P}$ analyzer with a Tinaquant CRP (Latex) highly sensitive immuno-turbidimetric assay (CRP latex HS, Roche kit, Roche Diagnostics, GmbH, Mannheim, Germany). Positive blood cultures were identified with the BACTEC FX automatic blood culture detection system (Becton Dickinson, Sparks, MD, USA) in the medical microbiology laboratory. The antimicrobial susceptibility of isolated strains was determined by the Phoenix Automated Microbiology System (BD Diagnostic Systems, Sparks, MD) in accordance with the European Committee on Antimicrobial Susceptibility Testing (EUCAST) criteria (13).

\section{Data analysis}

SPSS software 17.0 (SPSS, Chicago, IL) was used for statistical analysis. T-test and/or Mann-Whitney's U-test were used to compare non-parametric continuous variables in independent samples between groups, and chi-square test or Fisher's exact test for categorical variables. Results are given as mean and standard deviation and/or median (minimum-maximum) for continuous variables. Categorical variables were expressed as frequency and percentage distribution. Diagnostic screening tests to determine the cut-off for hemoglobin, platelet, CRP, RDW, MPV, PDW, and receiver operating characteristic (ROC) curve analysis were performed (sensitivity, specificity, positive predictive value, and negative predictive value) and $p$ value $<0.05$ was considered significant.

\section{RESULTS}

During the study period, 1,194 patients were admitted to our ICU and 336 patients were included in the study according to the inclusion criteria. Of the patients included in the study, 198 (58.9\%) patients died and 138 (41.1\%) were discharged after treatment (Figure 1). While the age, number of comorbid diseases, duration of MV, hospital stay, APACHE II score, and SOFA score of non-survivor patients were higher than those of survivors $(p<0.05)$, the results were similar in terms of gender and blood cultures ( $p>0.05$ ) (Table 1).

Among non-survivors and survivors, the results were similar in terms of WBC and MCV $(p=0.174$, $p=0.648$, respectively). RDW, MPV, PDW, and CRP were higher in the non-survivors group ( $p<0.001)$, and platelet and hemoglobin levels were significantly lower ( $p<0.001$ ) compared to the survivors (Table 2). ROC analysis was implemented for RDW, MPV, PDW, platelet, hemoglobin, and CRP for the prediction of mortality in elderly patients with sepsis. The

Figure 1. Flowchart of patient enrolment in the study

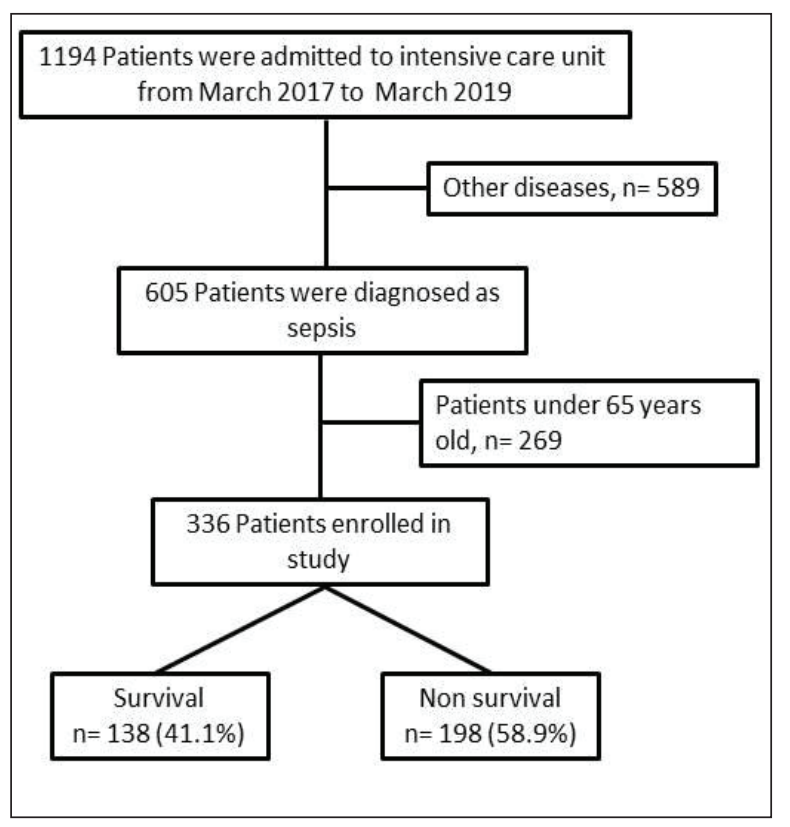


Table 1. Comparison of demographic and clinical features between survivors and non-survivors

\begin{tabular}{|l|c|c|c|}
\hline \multicolumn{1}{|c|}{ Variables } & $\begin{array}{c}\text { Survivors } \\
(\mathbf{n}=138)\end{array}$ & $\begin{array}{c}\text { Non-survivors } \\
(\mathbf{n}=198)\end{array}$ & P value \\
\hline Age, (years), a & $75.1 \pm 11.0$ & $82.1 \pm 7.9$ & $<0.001^{*}$ \\
\hline Male gender, $\mathrm{n}(\%)$ & $65(47.1)$ & $84(42.4)$ & 0.230 \\
\hline Comorbid disease, b & $2(0-5)$ & $3(0-6)$ & $<0.001^{*}$ \\
\hline APACHE II score, b & $19(11-34)$ & $24(15-47)$ & $0.001^{*}$ \\
\hline SOFA score, b & $7(3-16)$ & $10(6-21)$ & $<0.001^{\star}$ \\
\hline Duration of MV, (days), b & $4(1-41)$ & $7(1-45)$ & $0.001^{*}$ \\
\hline Hospital stay, (days), b & $11(5-69)$ & $28(1-144)$ & $<0.001^{*}$ \\
\hline Bacteremia, $\mathrm{n}$ (\%) & $31(22.4)$ & $58(29.2)$ & 0.291 \\
\hline
\end{tabular}

${ }^{a}$ mean \pm standard deviation, ${ }^{b}$ median (range), APACHE II: acute physiology and chronic health evaluation score, SOFA: sepsis-related organ failure assessment score, MV: mechanical ventilation

* Statistically significant $p$ values are highlighted.

Table 2. Comparison of laboratory variables between survivors and non-survivors

\begin{tabular}{|c|c|c|c|}
\hline Variables & $\begin{array}{c}\text { Survivors } \\
(n=138)\end{array}$ & $\begin{array}{c}\text { Non-survivors } \\
(n=198)\end{array}$ & $P$ value \\
\hline White blood cell count $\left(\times 10^{3} / \mu \mathrm{L}\right)$, a & $12.3 \pm 7.1$ & $13.1 \pm 11.4$ & 0.174 \\
\hline Hemoglobin (g/dL), a & $11.2 \pm 1.9$ & $9.0 \pm 1.1$ & $<0.001$ * \\
\hline Platelet $\left(\times 10^{3} / \mu \mathrm{L}\right)$, a & $298.6 \pm 129.6$ & $176.0 \pm 83.7$ & $<0.001^{\star}$ \\
\hline C-reactive protein $(\mathrm{mg} / \mathrm{L}),{ }^{a}$ & $78.4 \pm 67$ & $174.9 \pm 105.1$ & $<0.001^{*}$ \\
\hline Mean corpuscular volume (fL), a & $86.2 \pm 7.8$ & $85.9 \pm 6.2$ & 0.648 \\
\hline Red blood cell distribution width (\%), a & $15.7 \pm 2.3$ & $18.2 \pm 2.6$ & $<0.001^{\star}$ \\
\hline Mean platelet volume (fL), a & $9.4 \pm 1.1$ & $11.1 \pm 1.1$ & $<0.001^{*}$ \\
\hline Platelet distribution width (\%), a & $10.9 \pm 2.1$ & $15.0 \pm 2.9$ & $<0.001^{*}$ \\
\hline
\end{tabular}

a mean \pm standard deviation

*Statistically significant $p$ values are highlighted. 
cut-off value for mortality in elderly patients with sepsis was $\geq 16.3 \%$ for $R D W, \geq 10$ fL for MPV, $\geq 11.6 \%$ for PDW, $\leq 281 \times 10^{3} / \mu \mathrm{L}$ for platelet, $\leq 9.8 \mathrm{~g} / \mathrm{dL}$ for hemoglobin, and $86 \mathrm{mg} / \mathrm{L}$ for CRP $(p=0.0001)$. The results are presented in Figure 2 as area under curve $(A \cup C), p$ value, sensitivity, specificity, positive predictive value, and negative predictive value.

\section{DISCUSSION}

Our study is the first to evaluate the relationship between mortality and erythrocyte and platelet indices in elderly patients with sepsis in the ICU. According to our results, the high levels of MPW, PDW, RDW, and CRP and low platelet and hemoglobin counts were found to be significant indicators of mortality. High specificity, sensitivity, and positive predictive values increase the reliability of these tests' predictability. In the ROC analysis performed on the significance of these six hematological parameters for predicting mortality in elderly patients with sepsis when certain threshold values were exceeded, the AUC values were found to be between $0.7-0.9$ and had a moderate degree of accuracy (14). In addition to all laboratory findings, the increase in the num-

Figure 2. ROC curve for RDW, MPV, PDW, platelet, hemoglobin, and CRP, predicting mortality in the ICU
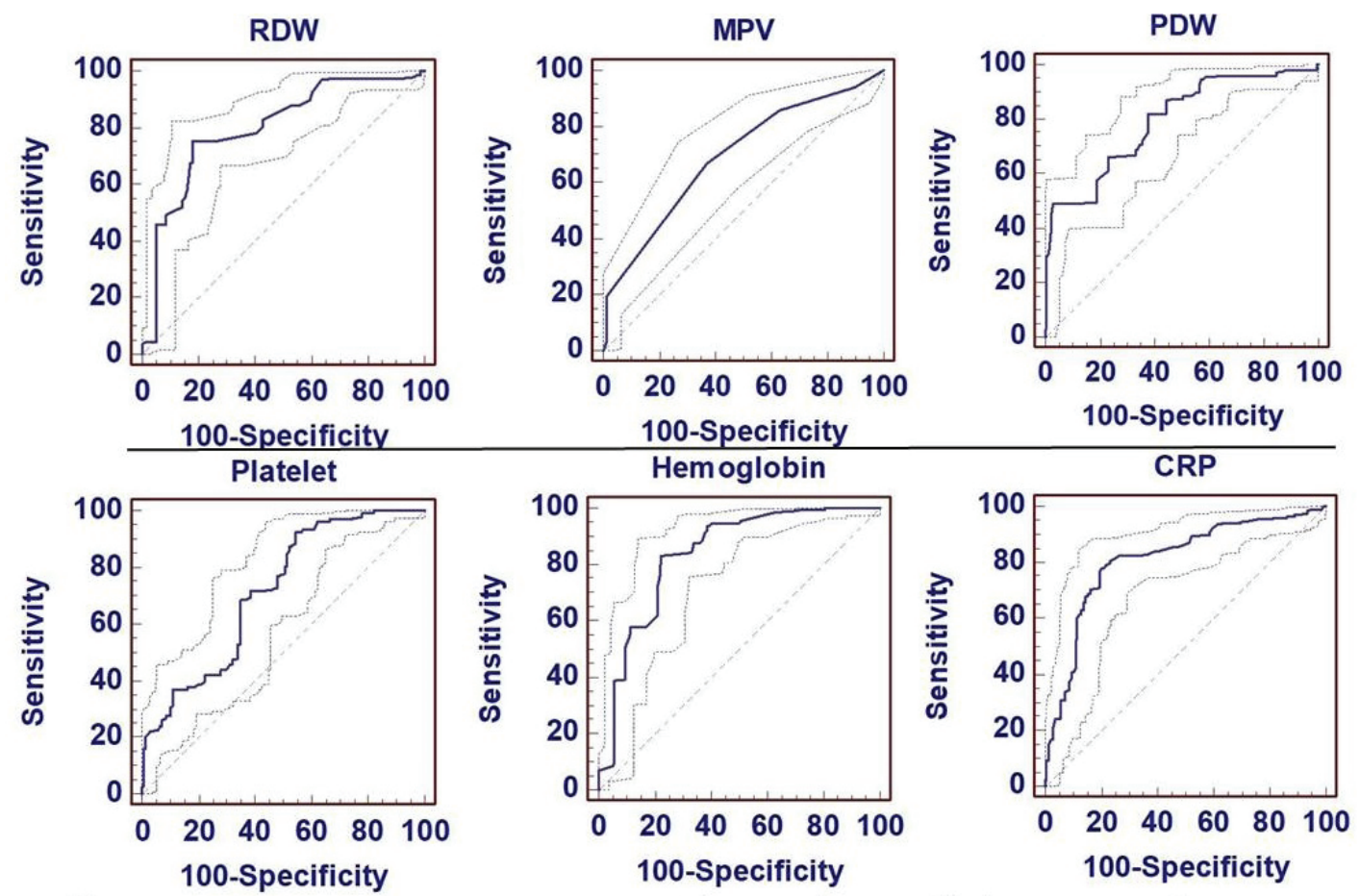

Variables

Red blood cell distribution width (RDW) (\%)

Mean platelet volume (MPV) (fL)

Platelet distribution width (PDW) (\%)

Platelet $\left(x 10^{3} / \mu \mathrm{L}\right)$

Hemoglobin (g/dL)

C-reactive protein (CRP) (mg/L)
AUC

\section{0-Specificity}

0.798

0.701

0.793

0.716

0.838

0.811
95\% Confidence p values interval

0.751-839

$0.650-0.751$

$0.746-0.835$

0.664-0.763

0.794-0.875

$0.765-0.852$
0.0001

0.0001

0.0001

0.0001

0.0001

0.0001
Cutofflevel Sensitivity Specificity PPV NPV $\begin{array}{llll}(\%) & (\%) & (\%) & (\%)\end{array}$ $\begin{array}{lllll}\geq 16.3 & 75 & 82 & 86 & 70\end{array}$ $\begin{array}{lllll}\leq 10 & 67 & 63 & 72 & 57\end{array}$ $\begin{array}{lllll}\leq 11.6 & 69 & 97 & 97 & 57\end{array}$ $\begin{array}{lllll}\leq 281 & 92 & 45 & 71 & 81\end{array}$ $\begin{array}{llllll}\leq 9.8 & 83 & 76 & 84 & 76\end{array}$ $\begin{array}{llllll}\geq 86 & 78 & 80 & 85 & 72\end{array}$

AUC: area under the curve, PPV: positive predictive values, NPV: negative predictive values, Roc: receiver operating characteristic 
ber of comorbid diseases increased APACHE-II and SOFA scores and also increased old age mortality (15-17).

Sepsis causes alterations in metabolism, a decrease in the level of 2.3 bisphosphoglycerate, erythrocyte deformability, and redistribution of membrane phospholipids on red blood cells (RBC) due to an irregular host response to infection. This dysregulation caused by sepsis is evident in erythrocytes, platelets, mitochondria, and microcirculation $(7,8)$. The change in RBC deformability is an early indicator of sepsis and reflects an existing inflammatory process size $(8,16)$. In septic patients characterized by a decrease in hemoglobin, MCV does not change during the first 24 hours of sepsis. Probably for this reason, in our study, MCV values were measured at the time of hospitalization, so the results were similar in the groups with and without mortality. In addition to the hemoglobin drop in sepsis, the oxygen dissociation curve shifts to the left, P50 (hemoglobin is $50 \%$ oxygen saturated) decreases, and the oxygen affinity of hemoglobin increases. Through these mechanisms, there is an increase in tissue hypoxia, organ failure, and mortality. Howev$\mathrm{er}$, this relationship grows more complicated in sepsis and is not fully understood (8).

The RDW parameter calculates (coefficient variation of RBC volume) and quantifies the RBC volume (anisocytosis) heterogeneity as the ratio of the standard deviation of the RBC volume to MCV. High RDW reflects the regulation disorder of erythrocyte homeostasis, which includes both impaired erythropoiesis and abnormal RBC survival (18). In cases of oxidative stress, such as sepsis, erythropoiesis is disrupted, anisocytosis increases, RBC membrane deformability changes circulation half-life and eventually increases RDW (19). Different results about the effects of RDW and CRP on mortality in patients with sepsis and the effect of MCV on mortality in patients with sepsis, have been shown in our study $(15-17,19)$. There are still some uncertainties about the importance of the RDW parameter in septic patients (20), however, and our study is important in terms of explaining the prognostic factor and mechanism behind RDW in elderly patients with severe sepsis. All these contradictory results show that more research is necessary to determine the relationship between cause and effect, sepsis pathophysiology, and patient outcomes with changes in RBC volume heterogeneity from sepsis (8).

MPV is another prognostic marker for septic shock and its level is higher in patients who die (6). Patrick et al. reports that high PDW levels, in addition to high MPV, had $95 \%$ and $79 \%$ specificity, respectively, in detecting bacteremia (21). In another study, higher MPV and PDW levels and lower platelet counts were found in sepsis patients compared to the control group. It has been reported that this situation is caused by greater cytokine production, endothelial damage, and bone marrow suppression in septic patients (7). In endotoxemia, the animal experiment was supported by decreasing platelets and increasing MPV and PDW (22). Our results were similar to previous studies with high MPV and PDW and low platelets used as sensitive markers to predict mortality in sepsis patients. As in our results, sometimes a decrease in the platelet count without thrombocytopenia may be associated with increased mortality (23). These results can be explained by the fact that coagulation and platelet activation/hyper-aggregation may occur in the early stage of sepsis, and the platelets transform their discoid shapes into global shapes during activation to obtain a larger surface (7). In addition to the physiologically increased platelet production process, rapid platelet production occurs after cytoplasmic rupture and megakaryocyte rupture in response to a sharp decrease in the platelet count. Thanks to this alternative mechanism, a high rate of MPV is rapidly released into the platelet blood flow. As a result, these large platelets become more active than small platelets (6). This explains the increase in MPW and RDW in sepsis patients and, thus, the increase in mortality. 
In our study, it was found that RDW $\geq 16.3 \%$, MPV $\geq 10 \mathrm{fL}, \mathrm{PDW} \geq 11.6 \%$, and platelet counts $\leq 281 \times 10^{3} /$ $\mu \mathrm{L}$ increased mortality in septic geriatric patients. Guclu et al. found that having PDW $>17.9 \%$, MPV $>8 \mathrm{fL}$, and platelet counts $\leq 155 \times 10^{3} / \mu \mathrm{L}$ predicted sepsis compared to the control group (7). Jiang et al. found that a PDW $>13.7 \%$ can be evaluated as a parameter for the indicator of 28-day mortality of sepsis patients in the ICU (17). In some studies, evaluating patients admitted to the ICU, different threshold values in the range of 8.9-11.6 (fL) were found in the use of MPV as an independent predictive indicator of mortality. These differences may be a result of the patients' ages and the heterogeneity of the diagnoses in the compared groups $(6,24)$. Our cut-off results were valid for geriatric septic patients in a single center. In our study, AUC was between 0.7-0.9 for MPW, PDW, RDW, platelet, hemoglobin, and CRP as an indicator of mortality in patients with sepsis in the ICU, and AUC was found to be valuable in the moderate accuracy level in mortality estimation (14). In some studies, using the above parameters as an indicator of mortality, AUC accuracy values were found to be similar to our results with moderate accuracy $(0.7-0.9)(6,7,19,24$, 25).

\section{Study limitations}

This study has potential limitations due to retrospective cohort analyzes, a single-center study, and unmeasured confounding variables. We did not have any data on pre-hospital laboratory values, previous hematological abnormalities, previous transfusion, blood cell morphology, hemolysis, reticulocytosis, bleeding, iron levels, and vitamin levels. This may have prevented us from identifying the cause and effect relationship in our patients and how these hematological abnormalities can lead to higher mortality. We focused on acceptance laboratory values to estimate mortality and were unable to determine whether changes in these parameters affect the risk of mortality during hospitalization.

\section{CONCLUSION}

To our knowledge, our study is the first to evaluate the relationship between erythrocyte and platelet indices mortality in geriatric septic patients in the ICU. Our results show that changes in erythrocyte and platelet indices can be used as significant parameters in predicting mortality in septic geriatric patients. All these indices are parameters that can be measured by routine blood count, which can be obtained cheaply and easily. Future studies are needed to determine whether changes in these parameters specifically reflect the underlying pathophysiology or only greater disease severity, to better identify the underlying pathophysiology in geriatric patients with sepsis, and to understand the relationship between abnormal hematopoiesis and outcomes.

\section{Conflict of Interest, Disclosure Statement}

The authors declare that they have no conflicts of interest. The authors have indicated they have no financial relationships relevant to this article to disclose

Ethical Approval: All procedures performed in this study involving human participants were in accordance with the ethical standards of the institutional and/or national research committee and with the 1964 Helsinki declaration and its later amendments or comparable ethical standards.

\section{Acknowledgement: none}




\section{REFERENCES}

1. Nasa $P$, Juneja D, Singh $O$, Dang $R$, Arora V. Severe sepsis and its impact on outcome in elderly and very elderly patients admitted in intensive care unit. J Intensive Care Med 2012;27(3):179-83. (PMID: 21436163).

2. Geyik FD, Yuce Y, Erkal KH, Cevik B, Citak N. The Use of Quick Sofa (Osofa) in elderly patients with sepsis in the intensive care unit. Turkish Journal of Geriatrics 2018;21(2):143-9. (DOI : 10.31086/tjgeri.2018240414).

3. Angus DC, Kelley MA, Schmitz RJ, White A, Popovich J Jr; Committee on Manpower for Pulmonary and Critical Care Societies (COMPACCS). Caring for the critically ill patient. Current and projected workforce requirements for care of the critically ill and patients with pulmonary disease: can we meet the requirements of an aging population? JAMA 2000;284(21):2762-70. (PMID:11105183).

4. Rowe $T$, Araujo KL, Van Ness PH, Pisani MA, Juthani-Mehta M. Outcomes of older adults with sepsis at admission to an intensive care unit. Open Forum Infect Dis 2016;3(1):ofw010. (PMID:26925430).

5. Martin GS, Mannino DM, Eaton S, Moss M. The epidemiology of sepsis in the united states from 1979 through 2000. N Engl J Med 2003;348(16):1546-54. (PMID:12700374).

6. Vardon-Bounes F, Gratacap MP, Groyer S, et al. Kinetics of mean platelet volume predicts mortality in patients with septic shock. PLoS One 2019;14(10):e0223553. (PMID: 31622365).

7. Guclu E, Durmaz Y, Karabay O. Effect of severe sepsis on platelet count and their indices. Afr Health Sci 2013;13(2):333-8. (PMID:24235932).

8. Bateman RM, Sharpe MD, Singer M, Ellis CG. The effect of sepsis on the erythrocyte. Int J Mol Sci 2017;18(9). pii: E1932. (PMID:28885563).

9. Singer M, Deutschman CS, Seymour CW, et al. The Third International Consensus Definitions for Sepsis and Septic Shock (Sepsis-3). JAMA 2016;315(8):80110. (PMID:26903338).

10. Rhodes A, Evans LE, Alhazzani W, et al. Surviving Sepsis Campaign: international guidelines for management of sepsis and septic shock: 2016. Crit Care
Med 2017;45(3):486-552. (PMID:28098591).

11. Knaus WA, Draper EA, Wagner DP, Zimmerman JE. APACHE II: a severity of disease classification system. Crit Care Med 1985;13(10):818-29. (PMID:3928249).

12. Vincent JL, Moreno R, Takala J, et al. The SOFA (Sepsis-related Organ Failure Assessment) score to describe organ dysfunction/failure. On behalf of the Working Group on Sepsis-Related Problems of the European Society of Intensive Care Medicine. Intensive Care Med 1996;22(7):707-10. (PMID:8844239).

13. European Committee on Antimicrobial Susceptibility Testing. Clinical breakpoints [internet]; 2016. Available from: http://www.eucast.org/clinical breakpoints. Accessed: 02.06.2020.

14. Akobeng AK. Understanding diagnostic tests 3: Receiver operating characteristic curves. Acta Paediatr 2007;96(5):644-7. (PMID:17376185).

15. Rayes HA, Vallabhajosyula S, Barsness GW, et al. Association between anemia and hematological indices with mortality among cardiac intensive care unit patients. Clin Res Cardiol 2020;109(5):616-27. (PMID:31535171).

16. Tonietto TA, Boniatti MM, Lisboa TC, et al. Elevated red blood cell distribution width at ICU discharge is associated with readmission to the intensive care unit. Clin Biochem 2018;55:15-20. (PMID:29550510).

17. Jiang $Y$, Jiang $F Q$, Kong $F$, et al. Inflammatory anemia-associated parameters are related to 28-day mortality in patients with sepsis admitted to the ICU: a preliminary observational study. Ann Intensive Care 2019;9(1):67. (PMID:31183575).

18. Salvagno GL, Sanchis-Gomar F, Picanza A, Lippi G. Red blood cell distribution width: A simple parameter with multiple clinical applications. Crit Rev Clin Lab Sci 2015;52(2):86-105. (PMID:25535770).

19. Kim S, Lee K, Kim I, Jung S, Kim MJ. Red cell distribution width and early mortality in elderly patients with severe sepsis and septic shock. Clin Exp Emerg Med 2015;2(3):155-61. (PMID:27752590).

20. Fontana $V$, Spadaro S, Bond $O$, et al. No relationship between red blood cell distribution width and micro- 
circulatory alterations in septic patients. Clin Hemorheol Microcirc 2017;66(2):131-41. (PMID:28128746).

21. Patrick $\mathrm{CH}$, Lazarchick J. The effect of bacteremia on automated platelet measurements in neonates. Am J Clin Pathol 1990;93(3):391-4. (PMID:2309660).

22. Yilmaz Z, Eralp O, Ilcol YO. Evaluation of platelet count and its association with plateletcrit, mean platelet volume, and platelet size distribution width in a canine model of endotoxemia. Vet Clin Pathol 2008;37(2):159-63. (PMID:18533914).

23. Moreau D, Timsit JF, Vesin A, et al. Platelet count decline: an early prognostic marker in critical- ly ill patients with prolonged ICU stays. Chest 2007;131(6):1735-41. (PMID:17475637).

24. Gençay I, Büyükkoçak Ü, Ateş G, Çağlayan O. Mean platelet volume and platelet distribution width as mortality predictors in intensive care unit. J Health Sci Med 2020;3(1): 51-5. (DOI: 10.32322/jhsm.643639).

25. Kandis H, Ozhan H, Ordu S, et al. The prognostic value of mean platelet volume in decompensated heart failure. Emerg Med J 2011;28(7):575-8. (PMID:20660896). 